\title{
Neurolin, the Goldfish Homolog of DM-GRASP, Is Involved in Retinal Axon Pathfinding to the Optic Disk
}

\author{
Heiko Ott, Martin Bastmeyer, and Claudia A. O. Stuermer \\ Department of Biology, University of Konstanz, 78457 Konstanz, Germany
}

\begin{abstract}
Young axons of new retinal ganglion cells (RGCs) in the continuously growing goldfish retina fasciculate with one another and their immediate forerunners on their path toward the optic disk and along the optic nerve. They express the immunoglobulin superfamily cell adhesion molecules (CAMs) neurolin (DMGRASP) and the L1-like E587 antigen. Repeated injections of Fab fragments from polyclonal antisera against neurolin (neurolin Fabs) into the eye of $3.4-\mathrm{cm}$-long and rapidly growing goldfish caused highly aberrant pathways of young RGC axon subfascicles in the dorsal retina. Many axons grew in circles and failed to reach the optic disk. In contrast, E587 Fabs, used in parallel experiments, disrupted the fascicles but did not interfere with the disk-directed growth. Neurolin Fabs also disturbed axonal fasciculation in vivo as well as in vitro but less
\end{abstract}

severely than E587 Fabs. Coinjections of both Fabs increased defasciculation of the dorsal axons in both aberrant and diskdirected routes. They also disrupted the order of young RGC axons in the optic nerve more severely than E587 Fabs alone. This demonstrates that the development of tight and orderly fascicles in the dorsal retina and in the optic nerve requires both E587 antigen and neurolin. More importantly, our results suggest an involvement of neurolin in RGC axonal guidance from the retinal periphery to the optic disk. Because disrupted fascicles and errant axon routes were found only in the dorsal retinal half, a cooperation with so-called positional markers may be conceived.

Key words: retinal ganglion cell axons; pathfinding errors; cell adhesion molecules; immunoglobulin superfamily; antibodies; intraocular injections
During development, the axons of retinal ganglion cells (RGCs) receive segment-specific guidance cues along the path to their visual targets. The first segment of their path is from the soma to the fissure and later to the optic disk at the retinal center. Several extracellular matrix proteins in the periphery of the differentiating RGCs and in the basal lamina overlying the retina have been implicated in the prevention of RGC axon growth in the wrong direction and in the promotion of their disk-directed migration (Halfter and Deiss, 1984; Brittis and Silver, 1995). Moreover, immunoglobulin superfamily (IgSF) cell adhesion molecules (CAMs) on the axonal surfaces are involved in guiding RGC axons along other axons (Pollerberg and Mack, 1994; Bastmeyer et al., 1995; Brittis and Silver, 1995; Brittis et al., 1995). Finally, long-range chemoattractive cues emanating from the disk have been postulated but not identified (Ramon y Cajal, 1972; Deiner et al., 1997). Once at the disk, axons leave the retina and enter the optic nerve head, responding to netrin-1 via a receptor complex that includes the IgSF CAM deleted in colon rectal cancer (DCC) (Deiner et al., 1997).

The retina of goldfish grows throughout life, adding new RGCs around its circumference (Johns, 1977; Easter et al., 1981, 1984). It thus represents a favorable system for studies on RGC axon pathfinding mechanisms. The axons of newborn RGCs fasciculate and navigate in association with their immediate forerunners to the optic disk (Easter et al., 1984; Bastmeyer et al., 1990; 1995; Vielmetter et al., 1991). They enter into the optic nerve head as one coherent bundle and remain together along most of their

\footnotetext{
Received Dec. 18, 1997; revised Feb. 17, 1998; accepted Feb. 23, 1998.

This work was supported by grants of the Deutsche Forschungsgemeinschaft to C.A.O.S. We thank Ulrike Binkle for technical assistance and Mary Anne Cahill for correction of the English.

Correspondence should be addressed to Claudia A. O. Stuermer, Department of Biology, University of Konstanz, D-78457 Konstanz, Germany.

Copyright (C) 1998 Society for Neuroscience $\quad 0270-6474 / 98 / 183363-10 \$ 05.00 / 0$
}

path to the optic tectum. In fish, IgSF CAMs are selectively expressed by the differentiating RGCs and their axons (Stuermer et al., 1992) and are believed to participate in axon growth along other axons. This has been confirmed in the case of the E587 antigen, a member of the L1 subfamily of IgSF CAMs (Giordano et al., 1997). Repeated injections of Fab fragments of a polyclonal E587 antiserum (E587 Fabs) into the eyes of rapidly growing goldfish (which acquire roughly 50,000 new RGCs over the 10 week experimental period) caused a delay in association of young axons into fascicles. Fascicles and individual axons intersected, and the fascicles reached the optic disk in a broadened front (Bastmeyer et al., 1995).

Growing goldfish RGC axons also carry neurolin on their surfaces (Paschke et al., 1992). Neurolin, the homolog of avian DM-GR ASP/SC-1/BEN consists of five Ig-like domains, a transmembrane, and an evolutionary highly conserved cytoplasmic domain (Brümmendorf and Rathjen, 1994). DM-GR ASP/SC-1/ BEN (Burns et al., 1991; Tanaka et al., 1991; Pourquié et al., 1992) mediates cell adhesion through homophilic interactions and promotes axon growth and fasciculation.

Our present in vivo and in vitro assays demonstrate that goldfish neurolin also contributes to axon fasciculation, but its effect is less pronounced than that of E587 antigen. In our in vivo assays we injected Fab fragments of a polyclonal antiserum against immunopurified neurolin (neurolin Fabs) into the eyes of rapidly growing goldfish. Under these conditions, RGC axons commit severe pathfinding errors and fail to maintain their disk-directed growth, suggesting that neurolin participates in intraretinal RGC axon guidance. Along with the disturbance of intraretinal fascicle order, which is caused by injections of neurolin and E587 Fabs, we also found defects in the arrangement of RGC axons in the optic nerve. 


\section{MATERIALS AND METHODS}

Animals. Common goldfish (Carrassius auratus; body length, 5-7 cm) obtained from a local supplier were used for protein isolation and neuronal tissue cultures. In vivo experiments were performed with juvenile goldfish from our breeding colony at the University of Konstanz. For these in vivo tests, groups of 10 individuals were kept in 1001 tanks at $22^{\circ} \mathrm{C}$ and fed twice a day to accelerate their growth. For intraocular injections of antibodies and optic nerve transection, fish were anesthetized in MS 222 (3-aminobenzoic acid ethyl ester; Sigma, St. Louis, MO) in compliance with animal welfare legislation.

Antibodies. Monoclonal antibody (mAb) E21 (Paschke et al., 1992) was used for immunoaffinity purification of neurolin as described. Immunopurified neurolin was used in functional in vitro assays as a substrate for axon growth and for immunizing a BALB-c mouse (as described previously) (Vielmetter et al., 1991) against neurolin from which mAb N518 was obtained. mAb N518 against neurolin and mAb E587 against E587 antigen (Vielmetter et al., 1991) were used to immunolabel and thus visualize young growing RGC axons in experiments in which polyclonal antibodies were used for functional in vivo and in vitro assays.

Polyclonal antibodies were produced by injecting rabbits subcutaneously with immunopurified E587 antigen (Bastmeyer et al., 1995) or neurolin (Laessing et al., 1994). Complete Freund's adjuvant was used for the first injection, and incomplete Freund's adjuvant was used for the three subsequent injections performed at 3 week intervals. Fab fragments were obtained from the IgG fraction of the antisera by a Papain digestion kit (Pierce, Rockford, IL). The specificity of Fab fragments against E587 antigen (in brief E587 Fabs) and neurolin (neurolin Fabs) was verified on Western blots with proteins from cell surface membranes of adult goldfish brains (Vielmetter et al., 1991; Paschke et al., 1992) and on cryostat sections of goldfish brains.

Substrate preparation. Sterilized coverslips were coated by exposing them to poly-L-lysine $(0.1 \mathrm{mg} / \mathrm{ml}$ in distilled water $)$ for $1 \mathrm{hr}$ at room temperature. Then they were rinsed in distilled water and air-dried. Immunopurified neurolin (30 $\mu \mathrm{l})$ (protein concentration $1 \mu \mathrm{g} / \mathrm{ml}$ in PBS) was introduced between two polylysine-coated coverslips at room temperature. After $2 \mathrm{hr}$, coverslips were washed in Leibowitz medium (L-15; Life Technologies; Gaithersburg, MD) and used immediately for outgrowth assays.

Goldfish retinal explants. In vitro functional assays were performed with regenerating retinal axons that readily extend from retinal explants when the fish optic nerve is transected 14-17 d before preparation. Goldfish retinal explants were prepared as described previously (Vielmetter and Stuermer, 1989). In brief, the retina was isolated and attached to a nylon filter (Hybond; Amersham, Braunschweig, Germany). Retina and filter were cut into strips $300 \mu \mathrm{m}$ wide and explanted, ganglion cell layer down, onto coated coverslips. Small metal blocks were placed on the ends of the segments to keep the retina in contact with the substrate. The cultures were kept in L-15 supplemented with $2 \%$ fetal calf serum (FCS) and $0.4 \%$ methyl cellulose at $22^{\circ} \mathrm{C}$.

Cerebellar neurons were obtained by dissociating pieces of the goldfish cerebellum essentially as described for glial cells (Bastmeyer et al., 1994). They were seeded onto polylysine-coated or polylysine-neurolin-coated coverslips and maintained under the same culture conditions as retinal mini-explants (see below). Axon density and length of axons were evaluated in relation to the substrate.

For quantitative outgrowth assays the isolated retinae were cut with a tissue chopper into small squares ("mini-explants") of $200 \times 200 \mu \mathrm{m}$. Mini-explants were seeded onto coated coverslips and grown in supplemented L- 15 at $22^{\circ} \mathrm{C}$ on either polylysine- or polylysine-neurolin-coated coverslips, and neurolin Fabs $(500 \mu \mathrm{g} / \mathrm{ml})$ diluted in L-15 were added. To keep mini-explants in place, an uncoated coverslip was placed on top, supported by two silicone spacers of $1.1 \mathrm{~mm}$ height. Culture medium filled the space between the two coverslips. After $48 \mathrm{hr}$ the number of axons per explant was counted with a $20 \times$ lens in an inverted microscope (Zeiss Axiovert) under phase contrast. Counts were made close to the explant where fasciculation is at a minimum.

To evaluate axon fasciculation, mini-explants were grown on polylysine-coated coverslips at $27^{\circ} \mathrm{C}$ in control medium (L-15, $10 \%$ FCS, $0.4 \%$ methylcellulose) and in medium containing either neurolin Fabs $(500 \mu \mathrm{g} / \mathrm{ml})$ or E587 Fabs $(500 \mu \mathrm{g} / \mathrm{ml})$. The mini-explants were photographed after $48 \mathrm{hr}$ in an inverted microscope under phase contrast with a $10 \times$ objective. Negatives (Agfa Pan 25) were digitized and contrastenhanced using metamorph software. A line was drawn around the explant at a distance of $250 \mu \mathrm{m}$ from the center of the explant, and axons intersecting this line were counted.
Initially, each assay was performed separately with explants from the dorsal and ventral retina because the growth of dorsal RGC axons in vivo was affected more severely by the Fabs than that of ventral axons. However, because no difference in their in vitro growth behavior was observed, data from both groups of axons were pooled and additional data were collected with explants regardless of their positional origin.

For videomicroscopy, neurolin Fabs $(500$ or $800 \mu \mathrm{g} / \mathrm{ml}$ ) diluted in medium (L-15, 10\% FCS, $0.4 \%$ methylcellulose) were added to the cultures when the retina strips were explanted onto polylysine-coated coverslips. Control cultures received the equivalent volume of Tris buffer. Growing axons extending from retinal strips in the presence of Fabs and controls were monitored with time-lapse videomicroscopy. Living axons were viewed with a $40 \times$ phase-contrast objective in an inverted microscope (Zeiss Axiovert) to which a camera was attached. The camera was connected to an image processor (Hamamatsu) and an S-VHS time-lapse recorder (Panasonic). To avoid continuous illumination, a shutter that opened every $5 \mathrm{sec}$ for $200 \mathrm{msec}$ was inserted into the light path. Four images were taken, averaged, and recorded. Axon growth was recorded in randomly selected fields for 3-6 hr. These video sequences served to evaluate the behavior of growth cones elongating on polylysine when they contacted another axon. Growth cones that changed their direction and elongated along the other axons for at least $1 \mathrm{hr}$ were counted as fasciculated as opposed to growth cones that continued to elongate on the polylysine substrate for at least $1 \mathrm{hr}$ after having contacted another axon. Growth cones in fascicles or on polylysine were also recorded in randomly selected fields for at least $1.5-2.0 \mathrm{hr}$, to measure their growth velocity.

In vivo functional assays. For in vivo functional assays, 6-month-old goldfish of approximately equal size (length, 3.3-3.9 cm; eye diameter, $3.5-4.0 \mathrm{~mm}$ ) were selected from our breeding colony, with 8-10 fish for each experimental group. Antibodies in Tris buffer $(10 \mathrm{~mm}, \mathrm{pH} 7.5)$ or buffer alone were injected into the vitreous chamber of the fish eye. The sclera and iris were penetrated with a syringe, and antibody solution was pressure-injected through the preformed hole using a glass micropipette connected to a picospritzer (Transjector, Eppendorf). Fish received injections twice a week for 10 weeks. The injected volume was $0.3 \mu \mathrm{l}$ during the first 3 weeks and was increased to $0.35 \mu l$ for the next 3 weeks and to $0.4 \mu \mathrm{l}$ for the last 4 weeks. This resulted in antibody concentrations of $0.3-0.4 \mathrm{mg} / \mathrm{ml}$ in the vitreous after each injection. Four groups of fish were injected as follows: (1) neurolin Fabs $(22 \mathrm{mg} / \mathrm{ml})$ through the temporal aspect of the left eye, right eye untreated; (2) neurolin Fabs (19 $\mathrm{mg} / \mathrm{ml}$ ) through the nasal aspect of the right eye, left eye untreated; (3) E587 Fabs $(20 \mathrm{mg} / \mathrm{ml})$ through the temporal aspect of the left eye, right eye untreated; and (4) both neurolin Fabs and E587 Fabs (22 and 23 $\mathrm{mg} / \mathrm{ml}$, respectively) through the temporal aspect of the left eye, with the right eye receiving an equal volume of buffer only. During these 10 weeks, the goldfish grew by $44 \%$ in length to a mean body length of 5.2 $\mathrm{cm}$ (range, $4.6-5.9 \mathrm{~cm}$ ). The injections had no negative effect on growth of the eye or retina. Both the injected and the control eye had the same size (mean eye diameter $4.4 \mathrm{~mm}$; range, $4-5 \mathrm{~mm}$ ), and both retinae had the same diameters at the end of the experiment.

Immunohistochemistry on retinal whole mounts. Eyes were isolated, the cornea and pigment epithelium were removed, and the retina (photoreceptor layer down) was attached to a nylon filter (Hybond, Amersham) by suction. In the first group of fish, the vitreous and the inner limiting membrane together with retinal blood vessels were removed carefully, but thereafter they were left intact to exclude the possibility that abnormal axonal growth patterns were obscured by ruptured axons that sometimes occur when vessels and basal lamina are removed. The retinae were fixed in methanol $\left(-20^{\circ} \mathrm{C}\right)$ for $5 \mathrm{~min}$ and treated with primary antibodies overnight $\left(\right.$ at $\left.4^{\circ} \mathrm{C}\right)$. Neurolin Fab-injected eyes were exposed to mAb N518 or E587, and E587 Fab-injected eyes were exposed to mAb N518. Control retinae (no antibodies injected) were exposed simultaneously to E587 Fabs and mAb N518 (10 and $100 \mu \mathrm{g} / \mathrm{ml}$, respectively, in PBS) or to neurolin Fabs and mAb E587 (10 and $50 \mu \mathrm{g} / \mathrm{ml}$, respectively, in PBS) to stain young axons. After three washes in PBS (10 min each) retinae were incubated in a mixture of dichlorotriazinyl amino fluorescein (DTAF)-coupled goat anti-rabbit antibodies and tetramethyl rhodamine isothiocyanate (TRITC)-coupled goat anti-mouse antibodies (both from Dianova, Hamburg, Germany). After $1 \mathrm{hr}$ at $37^{\circ} \mathrm{C}$, retinae were washed three times in PBS, coverslipped in Mowiol, and viewed in a Zeiss Axiophot using the appropriate filter sets.

Immunohistochemistry on optic nerve sections. The optic nerves of injected and control animals were separated from the eye, immersed in Tissue Tec (Miles, Elkhart, IN), and frozen. Cryostat transverse sections 
$(12 \mu \mathrm{m})$ were collected on uncoated coverslips, air-dried for 1-2 hr, and fixed in methanol $\left(-20^{\circ} \mathrm{C}\right)$. Sections from control fish were exposed simultaneously to mAb N518 and E587 Fabs or to mAb E17 and neurolin Fabs. Nerve sections from fish whose eyes were injected with neurolin Fabs were incubated with mAb E17, and nerve sections from fish whose eyes were injected with E587 Fabs were injected with mAb N518. Sections from fish injected with both Fabs were treated with either mAb E17 or mAb N518. After $10 \mathrm{hr}$, sections were washed three times in PBS (10 min each) and treated with DTAF-coupled goat anti-rabbit antibodies and TRITC-coupled goat anti-mouse antibodies for $1 \mathrm{hr}$ at $37^{\circ} \mathrm{C}$. Sections were rinsed in PBS, coverslipped with Mowiol, and viewed and photographed in a Zeiss Axiophot using the appropriate filter sets and a $20 \times$ lens. Photomicrographs in which the nerves were magnified $50 \times$ (and had diameters of $\sim 30 \times 20 \mathrm{~mm}$ ) were covered by a grid folio divided into squares of $3 \times 3 \mathrm{~mm}$, and grids containing profiles of labeled axons were marked. The cross-sectional area of the nerves was approximated by adding up the squares covering the nerve section. Squares overlying labeled axons were also added, and the values were expressed as a ratio.

For all figures in this study, original negatives were digitized with a Microtec ScanMaker, processed with Adobe Photoshop software, and printed on a Pictography 3000 printer (Fuji).

\section{RESULTS}

\section{In vitro functional analysis of neurolin}

To investigate whether neurolin shares the functional characteristics of its avian homolog (Burns et al., 1991) and promotes axon growth and axon fasciculation, we performed in vitro assays with regenerating neurolin-positive RGC axons extending from retinal explants.

On coverslips coated with polylysine alone (explants, $n=91$ ) or with polylysine and immunopurified neurolin (explants, $n=92$ ), retinal mini-explants extended a mean number of $13.5( \pm 11.5$ SEM) and 18.6 ( \pm 14.4 SEM) axons, respectively, after $48 \mathrm{hr}$. No difference exists between these values by statistical analysis (Kolmogorov-Smirnov two-group test). When axons on polylysineneurolin-coated coverslips grew in the presence of neurolin Fabs $(500 \mu \mathrm{g} / \mathrm{ml})$, no change in the mean number of axons $(17.8 \pm 14.7$ SEM) per explant $(n=103)$ was observed. The Fabs, however, had bound to the axons and the substrate: both were fluorescent after exposure to secondary rhodamine-coupled antibodies. Immunopurified neurolin failed to promote axon outgrowth in this test. Also, no increase in number or length of axons was noted with neurolin-immunopositive cerebellar neurons (data not shown), which contradicts the possibility that the failure to respond to immunopurified neurolin is RGC specific.

RGC axons tend to form fascicles on polylysine-coated coverslips (Fig. 1A), indicating that they prefer other axons over polylysine for their growth (Bastmeyer et al., 1995). In medium containing E587 Fabs $(500 \mu \mathrm{g} / \mathrm{ml})$, axons extending from miniexplants are conspicuously defasciculated (Fig. $1 C$ ). In the presence of neurolin Fabs $(500 \mu \mathrm{g} / \mathrm{ml})$, however, defasciculation is less evident (Fig. 1B). Mini-explants giving rise to numerous axons on all sides were selected for a semi-quantitative assessment. A line was drawn around the explant at $250 \mu \mathrm{m}$ distance from its center, and axons crossing the line were counted. Each line crossing, whether by a fascicle or a single axon, was counted as one, so that the number of crossings serves as an index for fasciculation. The mean number of line crossings was $76( \pm 16.9$ SEM) in E587 Fab-treated cultures (explants, $n=23$ ) as opposed to $52( \pm 12.1 \mathrm{SEM})$ in cultures with no Fabs added (explants, $n=$ $29)$ and $62( \pm 15.5$ SEM) in cultures treated with neurolin Fabs (explants, $n=27)$. Statistical analysis (Mann-Whitney $U$ test) indicates a significant difference between values in control and E587 Fab-treated cultures $(p \leq 0.001)$ and a difference between values in control and neurolin Fab-treated cultures $(p \leq 0.01)$ just
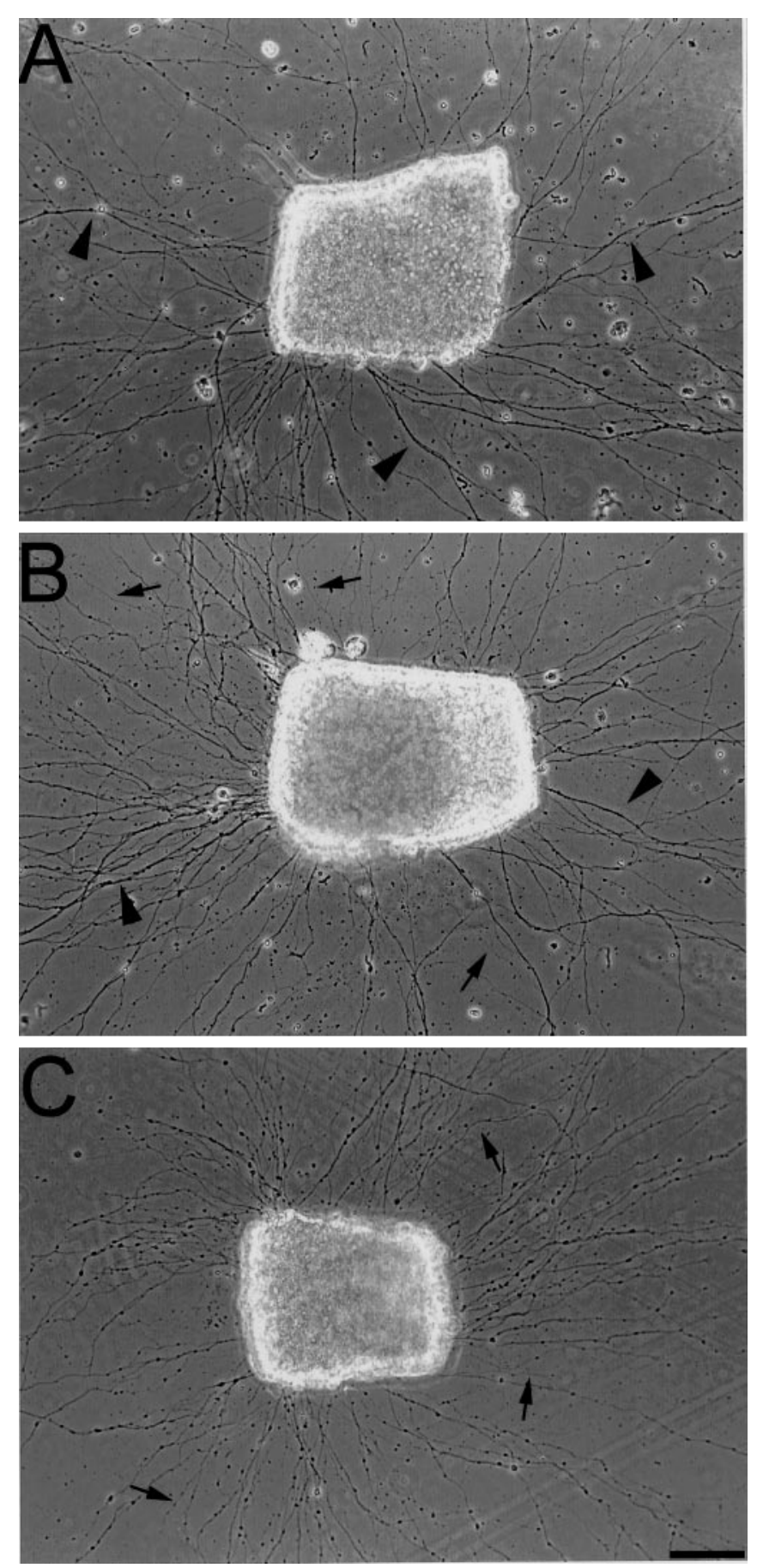

Figure 1. Neurolin and E587 Fab-induced axonal defasciculation in vitro. Phase-contrast micrographs of retinal mini-explants on polylysine are shown. $A$, In control medium the retinal axons grow preferentially in fascicles (arrowheads). The addition of neurolin Fabs $(B)$ results in a defasciculated growth pattern with many more single axons (arrows) in between fascicles (arrowheads). The number of individual axons (arrows) is further increased in the presence of E587 Fabs $(C)$. Scale bar, $50 \mu \mathrm{m}$.

at the limits of significance. The outcome of this analysis suggests a contribution of neurolin to RGC axon fasciculation that is less pronounced than that of E587 antigen.

Because direct recordings of individual growth cones is a more sensitive assay, we used time-lapse videomicroscopy to monitor the behavior and velocity of growth cones elongating on polyl- 
ysine and encountering another axon. Retinal strips from which the axons extend were raised in the presence of neurolin Fabs (500 $\mu \mathrm{g} / \mathrm{ml})$. Because earlier experiments (Bastmeyer et al., 1995) have shown that control antibodies (C 183) have no effect on axon fasciculation, the control cultures received no Fabs in the present tests. When they made contact with other axons, 54 growth cones continued to grow on polylysine in the presence of neurolin Fabs, and 26 (i.e., $33 \%$ ) fasciculated with other axons. In the control, 38 (of 74) growth cones continued to elongate on polylysine, and 36 (i.e., 48\%) migrated along another axon. Thus, when another axon is encountered, the preference of RGC growth cones to elongate along the other axon is reduced in the presence of neurolin Fabs, indicating that neurolin contributes to axon fasciculation.

The velocity with which an individual growth cone elongates along another axon was not affected by neurolin Fabs, being $55.4 \pm 19.8 \mu \mathrm{m} / \mathrm{hr}$ in the presence of neurolin Fabs (growth cones, $n=30$ ) and $51.0 \pm 15.2 \mu \mathrm{m} / \mathrm{hr}$ in the absence of neurolin Fabs (growth cones, $n=46$ ).

\section{In vivo functional analysis of neurolin in the retina}

To investigate whether and how neurolin contributes to the intraretinal path of young neurolin-immunoreactive RGC axons, rapidly growing goldfish received repeated injections of neurolin Fabs into one of their eyes through the nasal (10 fish) or temporal ( 9 fish) aspect of the eye. The control eyes received injections of the same volume of buffer or no injections, because nonimmune Fab injections had no effect on axon growth (Bastmeyer et al., 1995). Moreover, additional age- and size-matched fish $(n=9)$ were injected with E587 Fabs, which enabled us to compare the effects caused by the respective Fabs.

That the injected Fab fragments have access to the retinal axons and bind to them was confirmed in control fish receiving a single intraocular injection. Four days later, the retinae were prepared as whole mounts (with the overlying blood vessels left intact) and exposed to secondary antibodies. Young growing axons derived from the retinal margin exhibited immunoreactivity throughout their intraretinal path and in all retinal sectors. Thus, the injected Fab fragments have access to and are bound by young axons for at least $4 \mathrm{~d}$.

After repeated neurolin Fab injections, the orderly and direct growth toward the optic disk was disturbed in a substantial number of young RGC axons. In contrast to E587 Fabs that affected axon fasciculation but did not prevent axons from navigating to the optic disk, as shown in this study and in Bastmeyer et al. (1995), neurolin Fabs caused axons to deviate from their peripherocentral routes. These errant axons coexisted with axons associated in fascicles of normal orientation heading toward the disk and entering into the optic nerve head.

The errant axons exited from their fascicle of origin, often in small bundles, turned $180^{\circ}$, and coursed backward toward the retinal margin (Fig. 2). They eventually turned again, but instead of correcting their path they associated with other misrouted axons and bundles. This resulted in the formation of loops, circles, and multiple intersections, most often in areas between normal peripherocentrally oriented fascicles (Fig. 3). Axons in bundles of abnormal routes frequently left their counterparts to cross misrouted or normally oriented fascicles at various angles, or to end between the peripheral margin and optic disk (Fig. 3).

Axons committing these pathway errors coursed over substantial distances in fascicles of normal appearance and orientation before departing quite abruptly from their age-matched partners.
Such events were not always followed by a disoriented growth of axons because axons rejoined their original or another diskdirected fascicle (Fig. 2). This also occurred in cases in which axons grew obliquely across peripherocentrally directed fascicles and finally joined a more distant fascicle that they followed.

Less dramatic, but significant for neurolin Fab-affected retinae, was the apparent failure of smaller fascicles to merge after first encounter with neighboring fascicles. Instead, these fascicles crossed each other and later merged to form a thicker fascicle (Fig. 2A).

Figures 2 and $4 A$ show a further defect typical of neurolin Fab-injected retinae. Axons are loosely associated in bundles but do not adhere tightly to one another. This form of axon defasciculation qualitatively differs from that observed in E587 Fabtreated retinae at a comparable position where individual axons and small bundles cross each other and cross from one fascicle to another, and where axons are scattered more widely over this area of retina (Fig. $4 B$ ). Both differ from the control retina where axons are more tightly fasciculated (Fig. $4 C$ ) than in neurolin Fab-treated retinae, and they grow parallel to one another, in contrast to E587 Fab-treated retinae.

Surprisingly, all abnormal pathways in neurolin Fab-injected eyes were noted predominantly in the dorsal retina, but they were less prominent or absent from the ventral half (Fig. 5). This holds true for both groups of fish and was independent of whether the injection needle was introduced from the nasal or temporal pole of the eye. Thus, injections of neurolin Fabs can markedly disturb the normal growth behavior of young RGC axons over most of the retina. However, they seem to affect axons in the ventral retina only marginally or not at all.

From 19 neurolin Fab-injected eyes, 16 retinae were successfully prepared as whole mounts. All had defects, to variable extent, in their fascicle order. Misoriented axons (loops and circles) were observed in 10 retinae. Fascicle crossings followed by the reacquisition of a peripherocentral route through association with distant fascicles were found in 3 of these 10 and in an additional four retinae. All retinae contained fascicles in which axons failed to adhere tightly to one another (as illustrated in Figs. 2, 4A). Retinae of the uninjected and buffer-injected control eyes $(n=24)$ exhibited a normal fascicle order except for one (control of E587 Fab-injected eye) in which fascicles in the dorsonasal sector were disordered and had loops comparable to those seen after neurolin Fab injections.

Because abnormal pathways in neurolin Fab-injected eyes were noted in the dorsal retina but not in the ventral half, we examined whether this positional effect also occurs in the additional fish that received repeated E587 Fab injections over 10 weeks. Indeed, the order of young axons was normal in the ventral retina sector. The eight retina whole mounts successfully prepared from nine E587 Fab-injected eyes all exhibited a defasciculated growth pattern, as described earlier (Bastmeyer et al., 1995) in the dorsal retinal half. Features typical of neurolin Fab-injected eyes were not apparent after E587 Fab injections.

Thus, injections of E587 and neurolin Fabs can markedly disturb the normal growth behavior of young RGC axons over the dorsal retinal half, but they seem to affect axons in the ventral half only marginally or not at all. Although E587 Fabs disturb the development of orderly fascicles, and thus cause one form of axon defasciculation, they do not prevent their growth toward the optic disk or their exit into the optic nerve. Neurolin Fabs, however, prevent the tight binding of neighboring axons to one another and provoke the establishment of highly aberrant routes in which 

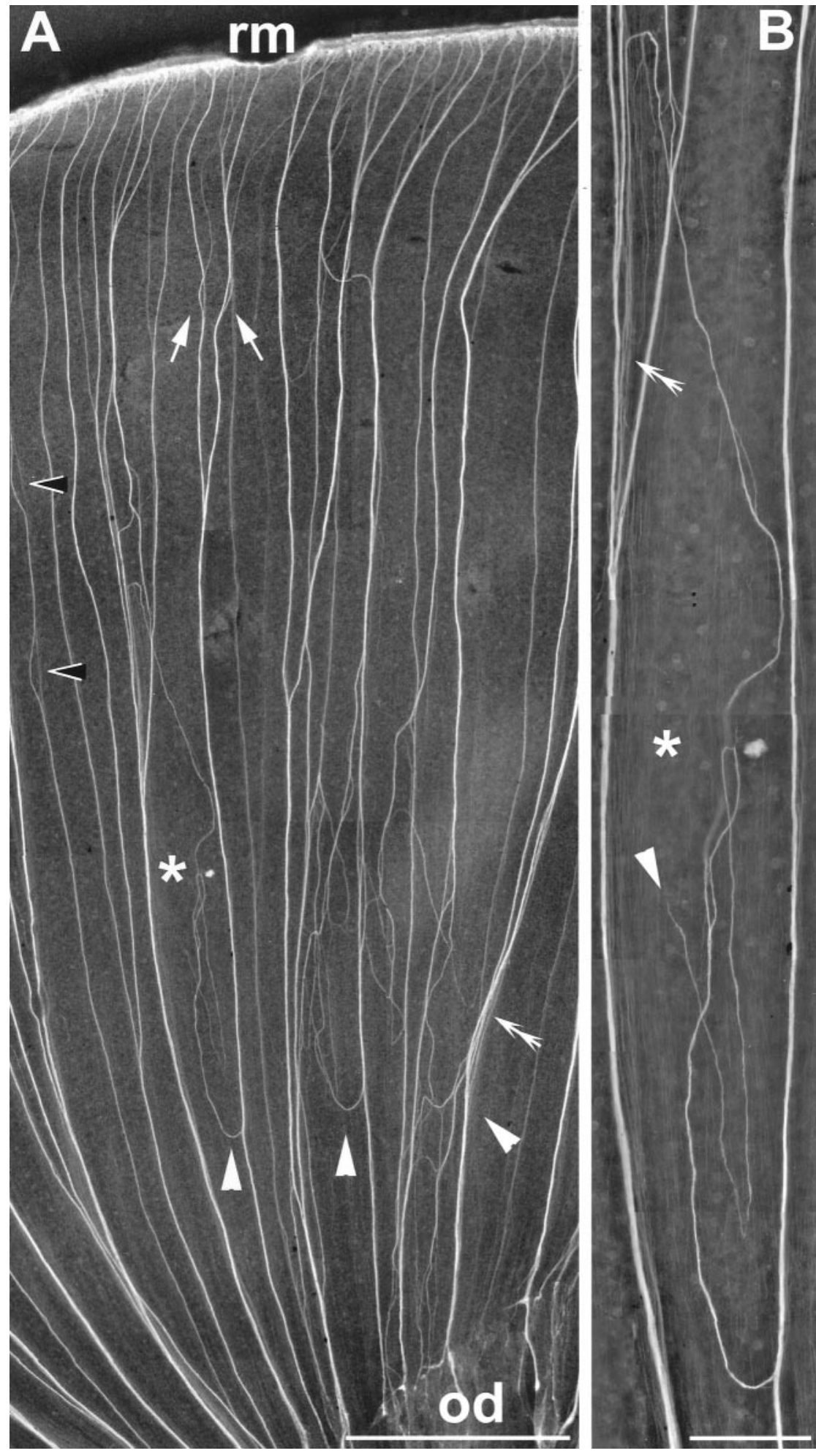

Figure 2. Aberrant axonal pathways 10 weeks after repeated intraocular neurolin Fab injections. In this dorsal segment of a flat-mounted retina, the young retinal axons on their path from their RGCs of origin at the retinal peripheral margin (top, $r m$ ) to the optic disk $(o d)$ are recognized by their immunofluorescence. $A$, Close to the margin, young axons meet and fasciculate with one another and normally travel in direct peripherocentral routes to the disk. Neurolin Fabs cause axonal pathway aberration: subfascicles depart from peripherocentrally oriented fascicles (white arrowheads), turn in the opposite direction, and establish loops and circles. Other abnormalities are fascicles failing to merge after first encounter (arrows) or failing to maintain their association (black arrowheads), as well as axons forming loose instead of tight bundles (double arrowheads). The region marked by the asterisk is shown enlarged in $B . B$, Higher magnification of the aberrant routes of subfascicles, which apparently have lost orientation and end (arrowhead) in an abnormal position. Portions of fascicles where axons have lost their tight association are marked by double arrowheads. Scale bars: $A, 500 \mu \mathrm{m} ; B, 100 \mu \mathrm{m}$. axons apparently lose orientation, fail to maintain their diskoriented growth, and end within the retina.

\section{Increased axonal defasciculation by neurolin Fabs and E587 Fabs in vivo}

We next explored whether the simultaneous injections of E587 and neurolin Fabs into one eye may cause additive defects in RGC axon growth. E587 Fabs $(23 \mathrm{mg})$ and neurolin Fabs $(22 \mathrm{mg})$ were mixed and injected into the left eye of 10 fish, equal in size to those described above and according to the same procedures. As shown in Figure 6, young RGC axons that grew in the presence of both Fabs were no longer associated in fascicles but were spread over the retinal surface. Many reached the optic disk and entered the optic nerve head, but axons growing in loops and away from the disk, which are typical effects of neurolin Fabs, were also identified. Individual axons and small bundles crossing other axons at various angles were frequent. All of this occurred 

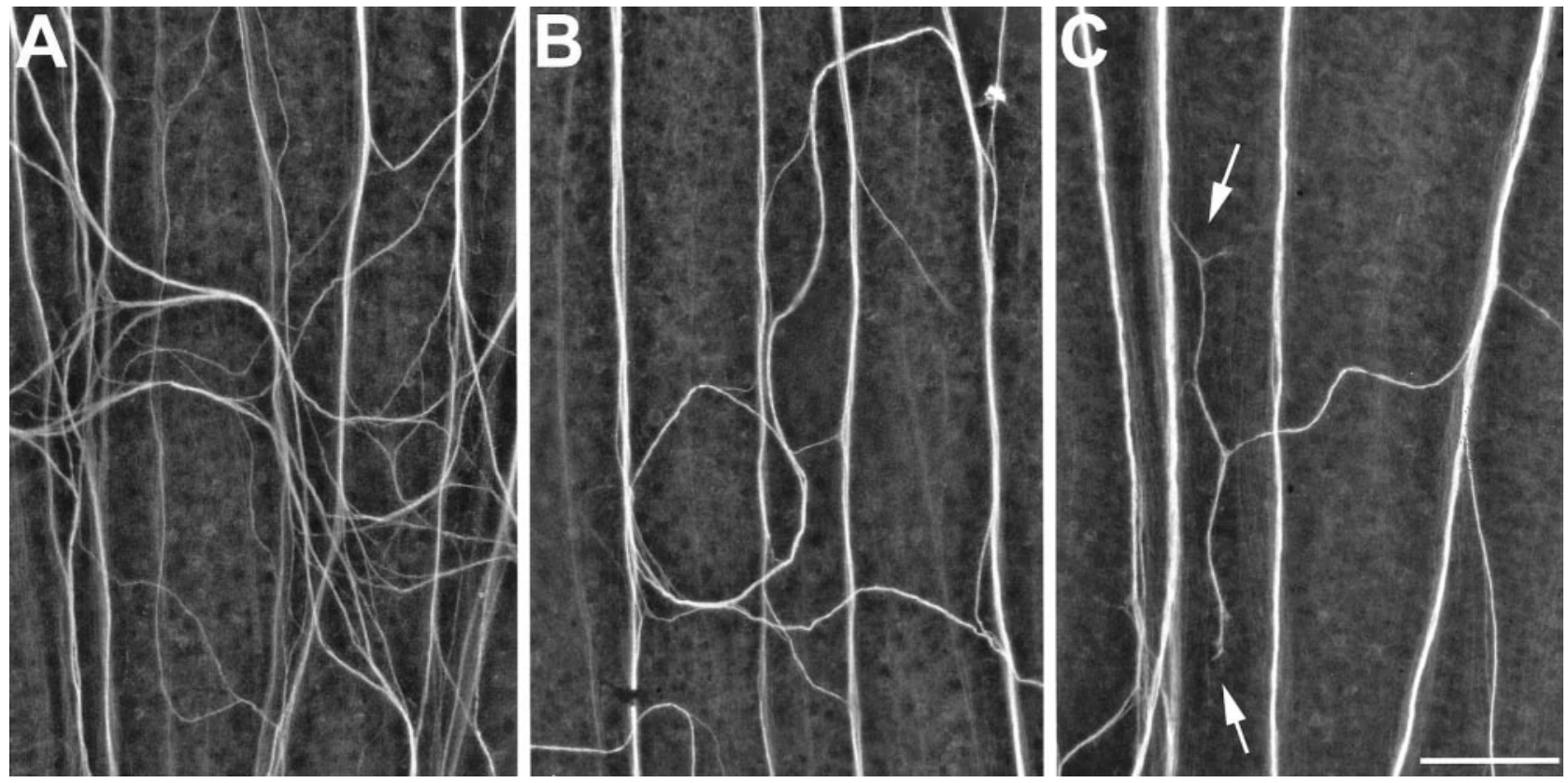

Figure 3. Examples of aberrant axonal routes from neurolin Fab-treated retinae. A, Young RGC axons no longer associate in distinct fascicles but course in various directions and form multiple intersections. $B$, Subfascicles separate from peripherocentrally oriented fascicles meet and establish abnormal circular routes or $(C)$ end in between fascicles (arrow) instead of traveling to the disk. All figures are oriented such that the retinal periphery is to the top and the disk to the bottom. Scale bar, $50 \mu \mathrm{m}$.
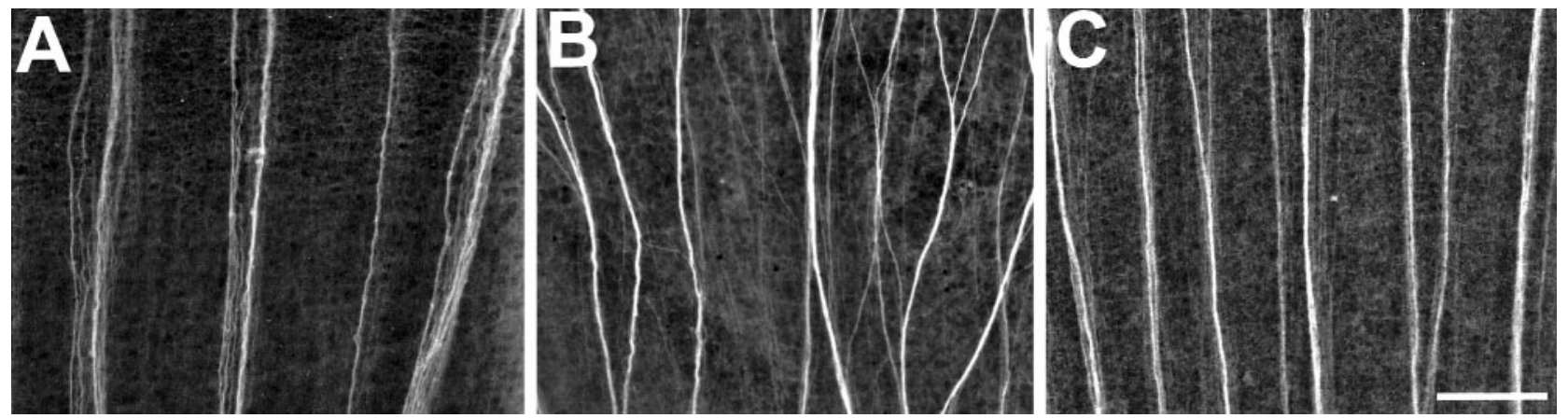

Figure 4. Comparison of axonal defasciculation caused by neurolin Fabs and E587 Fabs in vivo. A, Although in bundles, axons fail to fasciculate tightly in the presence of neurolin Fabs, i.e., the distance between near neighbors is increased as compared with the control $(C)$. B, E587 Fabs disrupt the fascicles and cause an increase in the number of subfascicles and single axons. Micrographs are taken from dorsal retina halves, roughly halfway between periphery and optic disk. Scale bar, $100 \mu \mathrm{m}$.

in the dorsal retina. Axons in the ventral retina again appeared rather normal.

\section{The position of young RGC axons in the optic nerve is affected after intraocular Fab injections}

Because both neurolin Fabs and E587 Fabs interfere with the formation of the normal fascicle order in the retina, both antibodies may also elicit abnormalities in the order of young axons in the optic nerve. Ideally, young growing axons associate into a coherent bundle in the ventral aspect of the optic nerve and next to axons of the preceding generation of RGC axons (Scholes, 1979; Easter et al., 1981; Bastmeyer et al., 1990; Stuermer, 1990). In reality, however, they are often divided into two or three distinct bundles (Fig. 7A). Optic nerves of neurolin Fab-injected eyes also have new RGC axons restricted to specific and coherent bundles (Fig. 7B). Nerves of E587 Fab-injected eyes and from eyes receiving both E587 and neurolin Fabs have many new axon bundles scattered over most of the cross sections of the nerves, in addition to bundles at the ventral edge of the nerves (Fig. 7C,D).

An estimate of the cross-sectional area occupied by young axons in control nerves (of control eyes) was obtained by the ratio of subfields containing labeled axons to subfields devoid of labeled axons (see Material and Methods). The distribution of labeled axons in nerves belonging to neurolin Fab-injected $(n=$ 6), E587 Fab-injected $(n=7)$, and neurolin plus E587 Fabinjected eyes $(n=5)$ were compared. Young axons in control nerves $(n=22)$ occupy between $23 \%( \pm 7 \%)$ of the crosssectional area of the nerve and on average $20 \%( \pm 14 \%)$ of the cross-sectional area in nerves of neurolin Fab-injected eyes. There is no difference between these values by statistical analysis. In comparison with normal nerves, young axons occupy statistically significant (Mann-Whitney $U$ test) larger areas of 50\% ( \pm $14 \% ; p=0.01)$ and $61 \%( \pm 4 \% ; p=0.001)$ in nerves of $\mathrm{E} 587$ Fab-injected eyes and after simultaneous injections of neurolin 


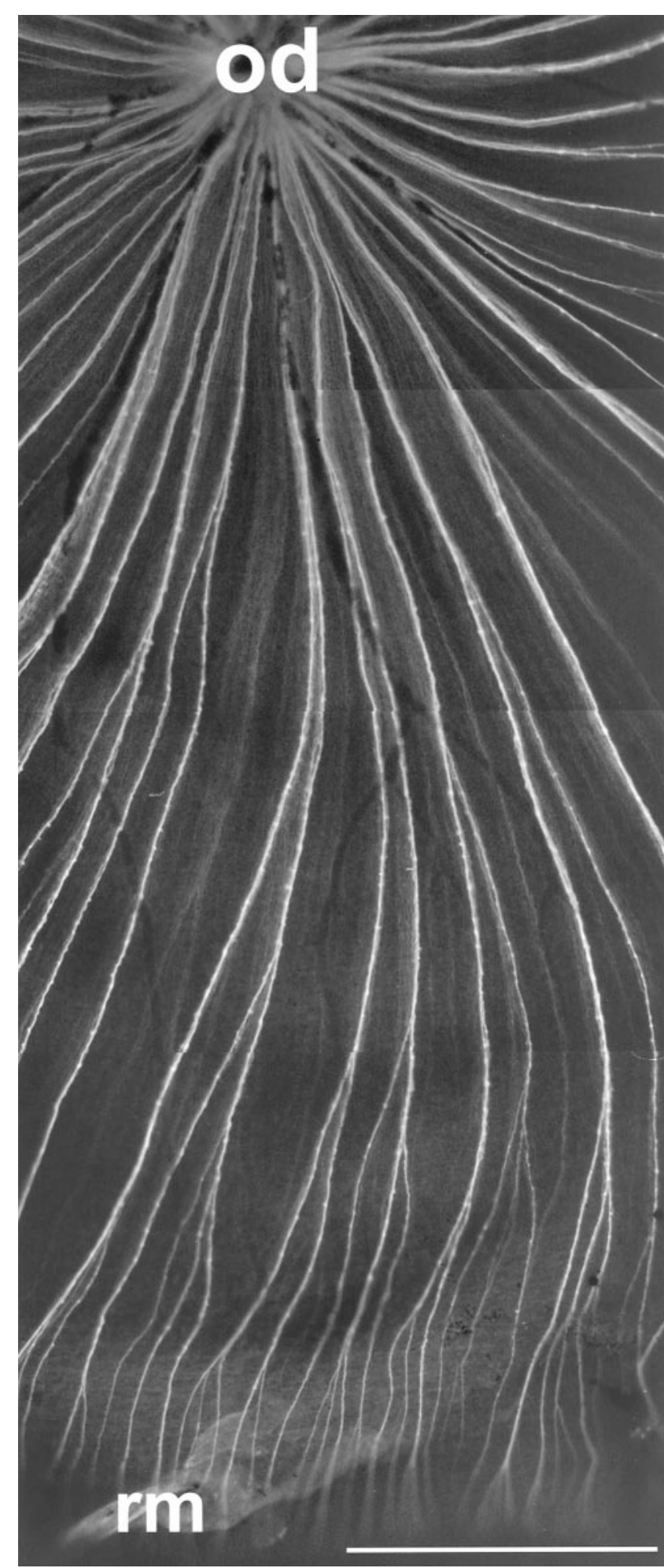

Figure 5. The fascicle order of young RGC axons in the ventral retina is normal. This sector of a retinal flat mount shows that young RGC axons in the ventral retina associate in orderly fascicles and course in direct routes to the optic disk $(o d)$, although they were exposed to neurolin Fabs during their growth. The retinal margin $(\mathrm{rm})$ and the new RGCs from which the young axons originate are out of focus. The curvature of the retina does not always allow it to flatten out entirely. Scale bar, $500 \mu \mathrm{m}$.
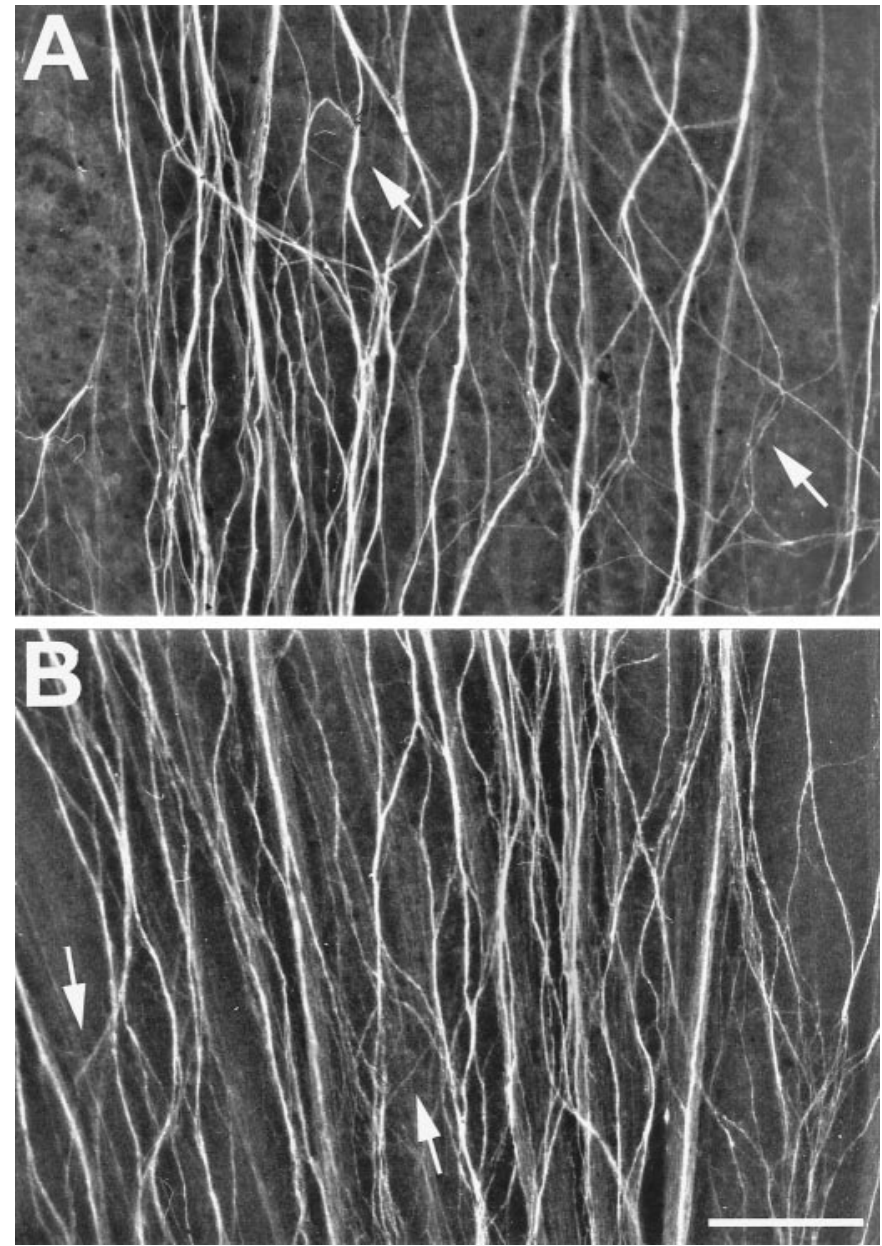

Figure 6. Increased axon defasciculation after coinjections of neurolin and E587 Fabs. $A, B$, Young RGC axons in the dorsal retina are spread over most of the retinal surface, and distinct fascicles are hardly recognizable. Highly aberrant routes are now established by small bundles or single axons (arrows) rather than by compact subfascicles. Scale bar, $100 \mu \mathrm{m}$.

and E587 Fabs, respectively. In conjunction with E587 Fabs, neurolin Fabs seem to increase the scattered distribution caused by E587 Fabs of young RGC axons over the nerve, but this is statistically insignificant.

These results demonstrate that E587 Fabs severely affect the mutual recognition of young axons that fail to associate into coherent bundles at defined positions in the nerve. Neurolin Fabs alone, causing defects in the disk-directed growth of RGC axons in the retina and having minor effects on axon fascicle formation, do not prevent the formation of coherent young RGC axon bundles in the nerve.

\section{DISCUSSION}

Neurolin in the goldfish retina appears to participate in the long-range axon pathfinding to the optic disk. When neurolin is blocked by binding with Fabs, neurolin-positive RGC axons no longer follow their normal disk-directed path. Many form loops, circles, or multiple intersections and fail to reach the optic disk. Blockade of the E587 antigen by E587 Fabs, on the other hand, disrupts the fascicles but not the disk-directed growth of the axons (Bastmeyer et al., 1995). This indicates that orderly fascicles are not a prerequisite for pathfinding and that the effects 
Figure 7. Disturbance of the order of young RGC axons in the optic nerve through neurolin Fabs and E587 Fabs. $A$, Immunolabeled young RGC axons in the optic nerve of a control (bufferinjected) eye course along the ventral aspect of the nerve, which is their normal position (arrowhead). B, Young RGC axons in a nerve of a neurolin Fab-injected eye are also correctly positioned at the ventral margin of the nerve (arrowhead) and in distinct fascicles at the edge of a large compact bundle in a more central location (arrow). C, After E587 Fab injections into the eye, young RGC axons are found at the ventral edge of the nerve (arrowhead) as well as scattered over the cross-sectional area of the nerve (examples marked by arrows). $D$, Young RGC axons in nerves of E587and neurolin Fab-injected eyes are also scattered widely over the cross-sectional area of the nerve (examples marked by arrows), but some occupy the normal position at the ventral edge of the nerve (arrowhead). Scale bar, $200 \mu \mathrm{m}$.
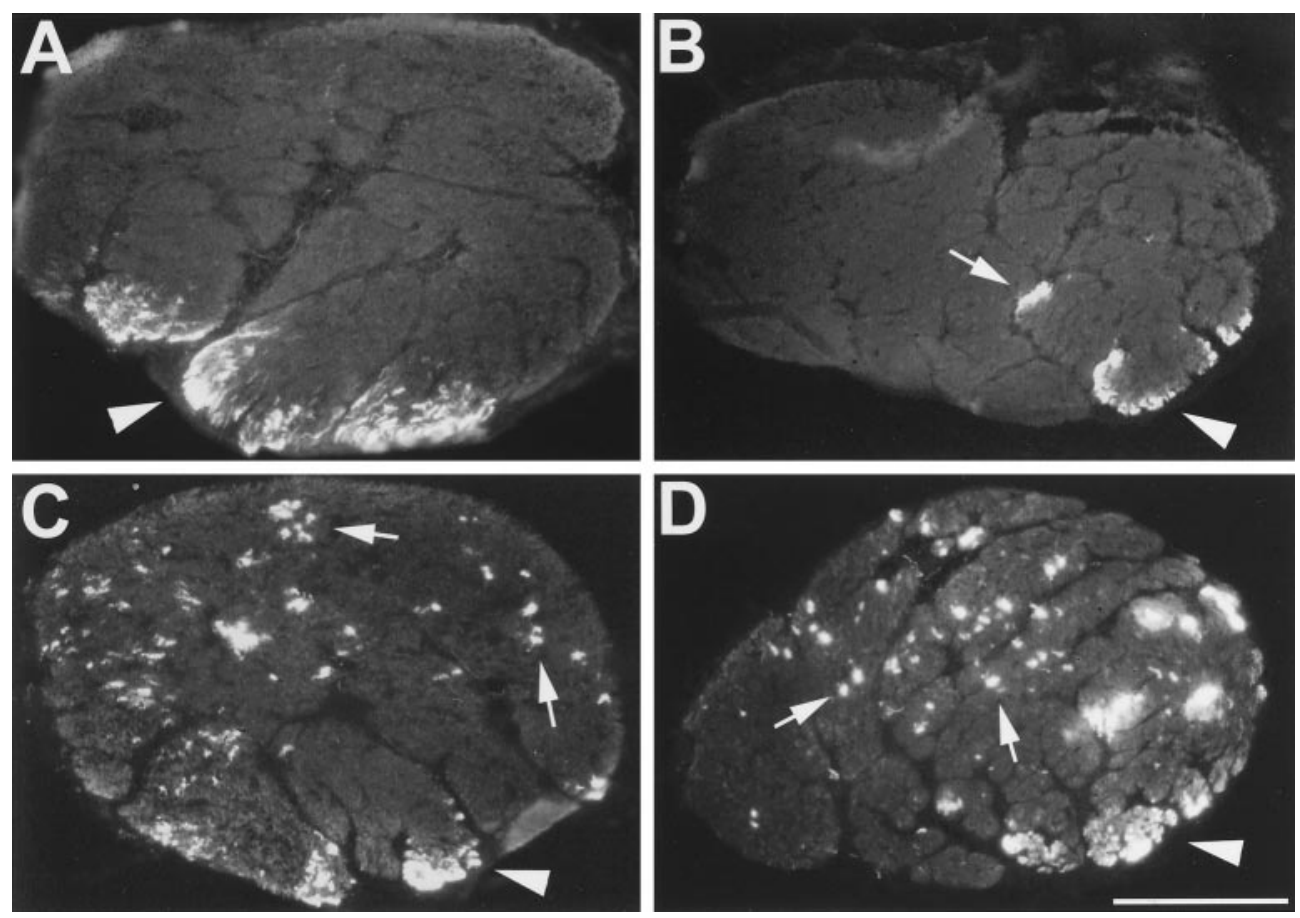

provoked by neurolin and E587 Fabs are specific. Neurolin Fabs interfere with axon fasciculation to a lesser extent than E587 Fabs, but both together cause young RGC axons to spread widely over the retinal surface. The disruption of retinal fascicles disturbs the order of young RGC axons in the optic nerve: they became scattered over the cross-sectional area of the nerve. This contrasts with the tight and orderly fascicles and peripherocentral orientation of the axons in control retinae and to the assembly of young axons into one or two coherent bundles at a defined position in the corresponding control nerves (Bastmeyer et al., 1990, 1995; Vielmetter et al., 1991).

Within the retina, pathway mistakes of young RGC axons are position dependent; they are not detected in the ventral retina. This may mean that molecules governing axonal pathfinding in the ventral retina are not identical with those contributing to pathfinding in the dorsal retina or that neurolin and E587 antigen function in the context of a complex molecular environment. It has been proposed that IgSF CAMs, and in particular L1 and NCAM, acquire signaling activities through their specific association with kinases, such as the FGF receptor (Doherty and Walsh, 1996; Hall et al., 1996). Moreover, L1 becomes phosphorylated by a member of the ephrin family of receptor tyrosine kinases (Zisch et al., 1997). Ephrins and their ligands are expressed unevenly in the retina and optic tectum and thereby may contribute to patterning in the retina and optic tectum (Nakamoto et al., 1996; Brennan et al., 1997; for review, see Drescher et al., 1997; Sefton and Nieto, 1997). They also contribute to axon fasciculation (Winslow et al., 1995; Caras, 1997). Thus, interactions between these molecules and CAMs expressed by axons may influence intraretinal axonal guidance. Such a functional link could account for the observed differences in axonal pathfinding in the dorsal and ventral retina after blockade of the CAMs investigated here. In two zebrafish mutants, defects in retinal axon pathfinding have been observed that concern dorsal RGC axons only (Trowe et al., 1996). This argues for the possibility that regional differences exist with regard to genes and molecules controlling axonal pathfinding within the retina.
All axons are fluorescently labeled after Fab injections and subsequent exposure to secondary antibodies, as shown in this study and in Bastmeyer et al. (1995). This excludes the existence of a diffusion barrier that might hinder Fab binding to the ventral retinal axons. The possibility remains, however, that the injected Fabs block the function of their respective antigen in the dorsal retina more efficiently than in the ventral half. In in vitro experiments, Fabs caused defasciculation of RGC axons from all areas of the retina, possibly because the Fabs have better access to antigen or because axons growing in culture are freed from the influence of positional factors.

The finding that neurolin contributes to axon fasciculation is consistent with reports on the function of its avian homolog (Burns et al., 1991). In vitro assays are not expected to reveal the function of neurolin in long-range axonal pathfinding outside of the tissue containing guidance information. Our present in vivo approach reveals a function of neurolin that has not been recognized for this CAM in earlier studies. Although DM-GRASP was shown to promote axon growth of GRASP-positive axons (Burns et al., 1991), we found no such effect of neurolin added to polylysine. We also found no neurolin-dependent increase in axon growth velocities when axons follow other axons. With similar assays, E587 antigen was shown to posses both of these properties (Bastmeyer et al., 1995). Thus, goldfish neurolin differs from E587 antigen and from its avian homolog DM-GRASP with respect to these functions.

With a single exception, the effects of neurolin and E587 Fab injections were not observed after control injections of nonimmune Fabs or buffer (Bastmeyer et al., 1995). This indicates that spontaneous axonal navigational errors can occur (Cook, 1982), but these are rare. More importantly, the injections have no noticeable negative influence on growth of the eye or retina, so that the procedure per se and the possible increase in intraocular pressure did not retard or otherwise change RGC axon development.

Under the premise that axons navigate to the optic disk under the influence of long-range guidance information (Ramon y Ca- 
jal, 1972; Deiner et al., 1997), deviation from the direct path to the optic disk and growth in the opposite direction when neurolin is blocked suggest that neurolin participates in axon guidance. Fabs derived from a polyclonal antiserum against neurolin block several sites of the protein. Therefore, the contribution of neurolin to long-range axonal guidance may reside in specific sites and its fasciculation-promoting function may reside in others. Preliminary results with domain-specific monoclonal antibodies support this view (C. Leppert, H. Diekmann, U. Laessing, M. Bastmeyer, and C. A. O. Stuermer, unpublished results). Also, $\mathrm{BEN}$ was demonstrated to interact with the scavenger receptor CD-6 (Bowen et al., 1997) and to mediate homophilic binding (Bowen et al., 1997; Skonier et al., 1996), and these functions were mapped to different Ig domains. Likewise, the Drosophila CAM, irre C-rst, a relative of DM-GRASP, mediates homophilic binding and has been proposed to interact with a heterophilic ligand (Schneider et al., 1995). This is consistent with the concept that neurolin has more than one function and contributes to axon fasciculation and guidance through different domains.

Although the location and identity of the guidance factors are presently unknown, a long-range chemoattractant centered around the optic disk (Ramon y Cajal, 1972; Deiner et al., 1997), a chemorepulsive activity in the periphery of the developing axons (Brittis and Silver, 1995), cues on glial endfeet and the basal lamina (Easter et al., 1984; Silver and Rutishauser, 1984; Halfter, 1996), or cues in guidance channels (Krayanek and Goldberg, 1981) are all conceivable alternatives. At the level of the individual axon, interference with neurolin function may weaken the tendency of growth cones to fasciculate with their age-related counterparts and forerunners and increase the probability that a growth cone departs from its fascicles. Growth cones in aberrant routes are followed by growth cones arriving later, probably because the forerunners, with several adhesion proteins (including E587 antigen) (Stuermer et al., 1992) on their surface, are still attractive substrates. If $\sim 50,000$ RGCs are being added over the 10 weeks (Bastmeyer et al., 1995) and if neurolin expression on the axons lasts for at least 4 weeks [an estimate derived from neurolin immunoreactivity on regenerating RGC axons (Paschke et al., 1992)], then ideally all these axons should be affected by neurolin Fabs during their growth. On the other hand, the presence of many CAMs allows young axons to reach the disk by tracking their forerunners, although the function of neurolin in the disk-directed growth is blocked. This could account for the fact that not all young axons can be disturbed during the 10 week period of neurolin Fab injections. Moreover, older axons that have already established routes to the optic disk are present, and they constitute a preformed path that young axons may choose to follow.

The degree of axon defasciculation resulting from coinjections of E587 and neurolin Fabs exceeds that achieved by each Fab alone. With regard to fasciculation, this suggests that E587 antigen compensates for, to some extent, the impaired function of neurolin and vice versa. When the mutual recognition and fasciculation of age-matched RGC axons in the retina is severely impaired, as is the case after blockade of E587 antigen alone [shown in this study and in Bastmeyer et al. (1995)] or in combination with blockage of neurolin, axons fail to associate into one or two distinct fascicles in the nerve. Under blockage of neurolin alone, young axons that exit the eye do form coherent bundles, but whether these bundles contain the normal number of axons remains to be analyzed. Also, whether axons occupying the correct ventral position in the nerve are derived from the ventral retina has not been examined. In goldfish (as well as in other fish) the order of RGC axons is determined by their retinal sectorial origin as well as by their age (Scholes, 1979; Easter et al., 1981; Vielmetter et al., 1991). The scattered distribution over the crosssectional area of the nerve that results from interfering with E587 antigen or both E587 antigen and neurolin functions shows that at least the age-related order of a substantial amount of axons is lost. Whether their order by retinal sectorial origin is also disturbed is not yet known.

It has recently been shown that axons in mice deficient in DCC and netrin-1 are appropriately guided to the optic disk but fail to enter into the optic nerve head (Deiner et al., 1997). In light of our present findings in goldfish, one might predict that homologs of neurolin may also contribute to the disk-directed growth in higher vertebrates, yet RGCs in embryonic rats did not express SC-1 mRNA until the day of birth (Jung et al., 1997). This contrasts with the situation in chick (Pollerberg and Mack, 1994) and embryonic fish (Laessing and Stuermer, 1996) in which neurolin mRNA and protein were detected on the first RGCs that differentiate and on all RGCs generated subsequently. This could imply that neurolin has similar functions for embryonic axons in these species.

\section{REFERENCES}

Bastmeyer M, Schlosshauer B, Stuermer CAO (1990) The spatiotemporal distribution of N-CAM in the retinotectal pathway of adult goldfish detected by the monoclonal antibody D3. Development 108:299-311.

Bastmeyer M, Jeserich G, Stuermer CAO (1994) Similarities and differences between fish oligodendrocytes and Schwann cells in vitro. Glia 11:300-314.

Bastmeyer M, Ott H, Leppert CA, Stuermer CAO (1995) Fish E587 glycoprotein, a member of the L1 family of cell adhesion molecules, participates in axonal fasciculation and the age-related order of ganglion cell axons in the goldfish retina. J Cell Biol 130:969-976.

Bowen MA, Bajorath J, D'Egidio M, Whitney GS, Palmer D, Kobarg J, Starling GC, Siadak AW, Aruffo A (1997) Characterization of mouse ALCAM (CD166): the CD6-binding domain is conserved in different homologs and mediates cross-species binding. Eur $\mathrm{J}$ Immunol 2:1469-1478.

Brennan C, Monschau B, Lindberg R, Guthrie B, Drescher U, Bonhoeffer F, Holder N (1997) Two Eph receptor tyrosine kinase ligands control axon growth and may be involved in the creation of the retinotectal map in the zebrafish. Development 124:655-664.

Brittis PA, Silver J (1995) Multiple factors govern intraretinal axon guidance: a time-lapse study. Mol Cell Neurosci 6:413-432.

Brittis PA, Lemmon V, Rutishauser U, Silver J (1995) Unique changes of ganglion cell growth cone behavior following cell adhesion molecule perturbations: a time-lapse study of the living retina. Mol Cell Neurosci 6:433-449.

Brümmendorf T, Rathjen F (1994) Cell adhesion molecules 1: immunoglobulin superfamily. London: Academic.

Burns FR, Von Kannen S, Guy L, Raper JA, Kamholz J, Chang S (1991) DM-GRASP, a novel immunoglobulin superfamily axonal surface protein that supports neurite extension. Neuron 7:209-220.

Caras IW (1997) A link between axon guidance and axon fasciculation suggested by studies of the tyrosine kinase receptor EphA5/REK7 and its ligand Ephrin-A5/AL-1. Cell Tissue Res 290:261-264.

Cook JE (1982) Errant optic axons in the normal goldfish retina reach retinotopic tectal sites. Brain Res 250:154-158.

Deiner MS, Kennedy TE, Fazelli A, Serafini T, Tessier-Lavigne M, Sretavan DW (1997) Netrin-1 and DCC mediate axon guidance locally at the optic disc: loss of function leads to optic nerve hypoplasia. Neuron 19:575-589.

Doherty P, Walsh FS (1996) CAM-FGF receptor interactions: a model for axonal growth. Mol Cell Neurosci 8:99-111.

Drescher U, Bonhoeffer F, Müller BK (1997) The Eph family in retinal axon guidance. Curr Opin Neurobiol 7:75-80.

Easter SS, Rusoff AC, Kish PE (1981) The growth and organization of the optic nerve and tract in juvenile and adult goldfish. J Neurosci 1:793-811. 
Easter SS, Bratton B, Scherer SS (1984) Growth-related order of the retinal fiber layer in goldfish. J Neurosci 4:2173-2190.

Giordano S, Laessing U, Ankerhold R, Lottspeich F, Stuermer CAO (1997) Molecular characterization of E587 antigen: an axonal recognition molecule expressed in the goldfish central nervous system. J Comp Neurol 377:286-297.

Halfter W (1996) Intraretinal grafting reveals growth requirements and guidance cues for optic axons in the developing avian retina. Dev Biol 177:160-177.

Halfter W, Deiss S (1984) Axon growth in embryonic chick and quail retinal whole mounts in vitro. Dev Biol 102:344-355.

Hall H, Williams EJ, Moore SE, Walsh FS, Prochiantz A, Doherty P (1996) Inhibition of FGF-stimulated phosphatidylinositol hydrolysis and neurite outgrowth by a cell-membrane permeable phosphopeptide. Curr Biol 6:580-587.

Johns PA (1977) Growth of the adult goldfish eye. III: Source of the new retinal cells. J Comp Neurol 176:343-357.

Jung M, Petrausch B, Stuermer CAO (1997) Axon-regenerating retinal ganglion cells in adult rats synthesize the cell adhesion molecule L1 but not TAG-1 or SC-1. Mol Cell Neurosci 9:116-131.

Krayanek S, Goldberg S (1981) Oriented extracellular channels and axonal guidance in the embryonic chick retina. Dev Biol 84:41-50.

Laessing U, Stuermer CAO (1996) Spatiotemporal pattern of retinal ganglion cell differentiation revealed by the expression of neurolin in embryonic zebrafish. J Neurobiol 29:65-74.

Laessing U, Giordano S, Stecher B, Lottspeich F, Stuermer CAO (1994) Molecular characterization of fish neurolin: a growth associated cell surface protein and member of the immunoglobulin superfamily in the fish retinotectal system with similarities to chick protein DM-GRASP/ SC-1/BEN. Differentiation 56:21-29.

Nakamoto M, Cheng HJ, Friedman GC, McLaughlin T, Hansen MJ, Yoon CH, O’Leary DDM, Flanagan JG (1996) Topographically specific effects of ELF-1 on retinal axon guidance in vitro and retinal axon mapping in vivo. Cell 86:755-766.

Paschke KA, Lottspeich F, Stuermer CAO (1992) Neurolin, a cell surface glycoprotein on growing retinal axons in the goldfish visual system, is reexpressed during retinal axonal regeneration. $\mathrm{J}$ Cell Biol $117: 863-875$.

Pollerberg GE, Mack TGA (1994) Cell adhesion molecule SC1/ DMGRASP is expressed on growing axons of retina ganglion cells and is involved in mediating their extension on axons. Dev Biol 165:670-687.

Pourquié O, Corbel C, Le Caer J-P, Rossier J, Le Douarin NM (1992) BEN, a surface glycoprotein of the immunoglobulin superfamily, is expressed in a variety of developing systems. Proc Natl Acad Sci USA 89:5261-5265.
Ramon y Cajal S (1972) The structure of the retina (English edition). Springfield, IL: Charles C. Thomas.

Schneider T, Reiter C, Eule E, Bader B, Lichte B, Nie Z, Schimansky T, Ramos RGP, Fischbach KF (1995) Neural recognition in the optic lobe of Drosophila: restricted expression of the IrreC-rst protein is required for normal axonal projections of columnar visual neurons. Neuron 15:259-271.

Scholes J (1979) Nerve fibre topography in the retinal projection to the tectum. Nature 278:620-624.

Sefton M, Nieto MA (1997) Multiple roles of Eph-like kinases and their ligands during development. Cell Tissue Res 290:243-250.

Silver J, Rutishauser U (1984) Guidance of optic axons in vivo by a preformed adhesive pathway on neuroepithelial endfeet. Dev Biol 106:485-499.

Skonier JE, Bowen MA, Emswiler J, Aruffo A, Bajorath J (1996) Recognition of diverse proteins by members of the immunoglobulin superfamily: delineation of the receptor binding site in the human CD6 ligand ALCAM. Biochemistry 35:12287-12291.

Stuermer CAO (1990) Target recognition and dynamics of axonal growth in the retinotectal system of fish. Neurosci Res 8[Suppl 13]:S1-S10.

Stuermer CAO, Bastmeyer M, Bähr M, Strobel G, Paschke K (1992) Trying to understand axonal regeneration in the CNS of fish. J Neurobiol 23:537-550.

Tanaka H, Matsui T, Agata A, Tomura M, Kubota I, McFarland KC, Kohr B, Lee A, Phillips HS, Shelton DL (1991) Molecular cloning and expression of a novel adhesion molecule, SC1. Neuron 7:535-545.

Trowe T, Klostermann S, Baier H, Granato M, Crawford AD, Grunewald B, Hoffmann H, Karlstrom RO, Meyer SU, Müller B, Richter S, Nüsslein-Volhard C, Bonhoeffer F (1996) Mutations disrupting the ordering and topographic mapping of axons in the retinotectal projection of the zebrafish, Danio rerio. Development 123:439-450.

Vielmetter J, Stuermer CAO (1989) Goldfish retinal axons respond to position-specific properties of tectal cell membranes in vitro. Neuron 2:1331-1339.

Vielmetter J, Lottspeich F, Stuermer CAO (1991) The monoclonal antibody E587 recognizes growing (new and regenerating) retinal axons in the goldfish retinotectal pathway. J Neurosci 11:3581-3593.

Winslow JW, Moran P, Valverde J, Shih A, Youan JQ, Wong SC, Tsai SP, Goddard A, Henzel WJ, Hefti F, Beck KD, Caras IW (1995) Cloning of AL-2, a ligand for an Eph-related tyrosine kinase receptor involved in axon bundle formation. Neuron 14:973-981.

Zisch AH, Stallcup WB, Chong LD, Dahlin-Huppe K, Voshol J, Schachner M, Pasquale EB (1997) Tyrosine phosphorylation of L1 family adhesion molecules: implication of the Eph kinase Cek5. J Neurosci Res 47:655-665. 